\title{
Extremal Graphs Having No Stable Cutsets
}

\author{
Van Bang Le \\ Institut für Informatik \\ Universität Rostock \\ Rostock, Germany \\ le@informatik.uni-rostock.de
}

\author{
Florian Pfender \\ Department of Mathematics and Statistics \\ University of Colorado at Denver \\ Denver, CO, U.S.A. \\ Florian.Pfender@ucdenver.edu
}

Submitted: Jul 4, 2012; Accepted: Feb 6, 2013; Published: Feb 18, 2013

Mathematics Subject Classifications: 05C35, 05C40, 05C69, 05C75

\begin{abstract}
A stable cutset in a graph is a stable set whose deletion disconnects the graph. It was conjectured by Caro and proved by Chen and Yu that any graph with $n$ vertices and at most $2 n-4$ edges contains a stable cutset. The bound is tight, as we will show that all graphs with $n$ vertices and $2 n-3$ edges without stable cutset arise recursively glueing together triangles and triangular prisms along an edge or triangle. As a by-product, an algorithmic implication of our result will be pointed out.
\end{abstract}

Keywords: stable cutset; independent cutset; fragile graph; extremal graph

\section{Introduction}

All graphs considered are finite and have no loops or multiple edges. For a graph $G=$ $(V(G), E(G))$ with vertex set $V(G)$ and edge set $E(G)$, write $|G|=|V(G)|$ and $\|G\|=$ $|E(G)|$. A stable set (or an independent set) in $G$ is a set of pairwise non-adjacent vertices. A cutset (or separator) of $G$ is a set $S$ of vertices such that $G-S$ is disconnected. A stable cutset in $G$ is a cutset of $G$ which is also a stable set. Throughout this paper, we consider the empty set as stable. So in particular, every disconnected graph contains a stable cut set. It is naturally expected that graphs with few edges would have stable cutsets. Indeed, the following theorem was conjectured by Caro and proved by Chen and $\mathrm{Yu}$.

Theorem 1 ([4]). Let $G$ be a graph with $\|G\| \leqslant 2|G|-4$. Then $G$ contains a stable cutset.

Small stable cutsets are discussed in [3], and algorithmic and complexity aspects of stable cutsets are discussed in $[5,1,7,8,9]$. The importance of stable cutsets in connection 
to perfect graphs are demonstrated in $[6,11]$. In [2] it is noted that graphs containing stable cutsets play a role in some decomposition algorithms.

Actually, Chen and Yu proved the following stronger result.

Theorem 2 ([4]). Let $G$ be 2-connected a graph with $\|G\| \leqslant 2|G|-4$. Then for every vertex $x \in V(G)$, there is a stable cutset not containing $x$.

This implies immediately the following corollary; a vertex $x$ is a cut vertex if $\{x\}$ is a cutset.

Corollary 3. Let $G$ be a graph with $\|G\| \leqslant 2|G|-4$, and $x \in V(G)$. Unless $x$ is the unique cut vertex in $G$, there is a stable cutset not containing $x$.

The bound in Theorem 1 is tight. In the next section we describe all graphs with $n$ vertices and $2 n-3$ edges that have no stable cutset (Theorem 5 ). In the last section we will point out an algorithmic implication of our result.

Notation and definitions. Let $G$ be a graph. The complement of $G$ is written $\bar{G}$. The neighborhood of a vertex $v$ in $G$, denoted by $N_{G}(v)$, is the set of all vertices in $G$ adjacent to $v$; if the context is clear, we simply write $N(v)$. Set $\operatorname{deg}(v)=|N(v)|$, the degree of the vertex $v$. For a subset $W \subseteq V(G), N(W)=\bigcup_{w \in W} N(w) \backslash W$, and $G[W]$ is the subgraph of $G$ induced by $W$; write $G-W=G[V(G) \backslash W]$ and $G-w=G-\{w\}$. Given another graph $H$, an $H$-cutset $S$ of $G$ is a cutset such that $G[S]$ is isomorphic to $H$, while a $k$-cutset is a $k$-element cutset. An edge cut of $G$ is a set $M$ of edges such that $G-M=(V(G), E(G) \backslash M)$ is disconnected. A matching cut in $G$ is an edge cut of $G$ that is also a matching.

$P_{k}$ stands for the path with $k$ vertices and $k-1$ edges, $C_{k}$ is the cycle with $k$ vertices and $k$ edges. A complete graph with $k$ vertices is denoted $K_{k} ; K_{k}^{-}$is $K_{k}$ minus an edge. The $K_{3}$ is also called a triangle and the $\overline{C_{6}}$ is also called a triangular prism; see Figure 1.

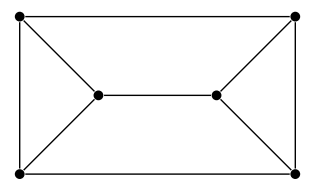

Figure 1: The triangular prism $\overline{C_{6}}$.

We will make use of the following well-known graph operation. A clique in a graph is a set of pairwise adjacent vertices. Let $G_{1}, G_{2}$ be disjoint graphs which each have nonempty cliques $Q_{1}$, respectively, $Q_{2}$ of the same size. A graph obtained from $G_{1}$ and $G_{2}$ by first choosing a bijection $f: Q_{1} \rightarrow Q_{2}$ and then identifying each $x$ in $Q_{1}$ with $f(x)$ in $Q_{2}$ is said to arise from $G_{1}$ and $G_{2}$ by clique identification. If the chosen cliques have two, respectively, three vertices, we also speak of edge identifications, respectively, triangle identifications. Finally, for convenience, we consider $G_{1}$ and $G_{2}$ as induced subgraphs of the graph arising from $G_{1}$ and $G_{2}$ by clique identification. Thus, a graph $G$ arises from two graphs by clique identification if and only if there exist induced subgraphs $G_{1}$ and $G_{2}$ in $G$ such that $G=G_{1} \cup G_{2}$ and $G_{1} \cap G_{2}$ is a clique. 


\section{The Result}

Let $\mathcal{G}_{s c}$ be the the class of graphs one gets by recursively glueing together triangles and triangular prisms along an edge or triangle. More precisely,

1. $K_{3} \in \mathcal{G}_{s c}$ and $\overline{C_{6}} \in \mathcal{G}_{s c}$.

2. If $G_{1}, G_{2} \in \mathcal{G}_{s c}$ and $G$ is obtained from $G_{1}$ and $G_{2}$ by edge identification, then $G \in \mathcal{G}_{s c}$.

3. If $G_{1}, G_{2} \in \mathcal{G}_{s c}$ and $G$ is obtained from $G_{1}$ and $G_{2}$ by triangle identification, then $G \in \mathcal{G}_{s c}$.

Notice that we may restrict to $G_{2} \in\left\{K_{3}, \overline{C_{6}}\right\}$ in the above definition without changing the class $\mathcal{G}_{s c}$, which effects the complexity of the algorithm considered in the last section of the paper.

Proposition 4. Any graph $G \in \mathcal{G}_{s c}$ has $\|G\|=2|G|-3$ edges and no stable cutset.

Proof. The statement is obvious for triangles and triangular prisms. Let $G$ arise from $G_{1}, G_{2} \in \mathcal{G}_{s c}$ by edge or triangle identification, and write $G=G_{1} \cup G_{2}$ with clique $Q=G_{1} \cap G_{2}$ of size two or three. Then

$$
|G|=\left|G_{1}\right|+\left|G_{2}\right|-|Q| \text { and } \quad\|G\|=\left\|G_{1}\right\|+\left\|G_{2}\right\|-\|Q\|,
$$

and hence, by induction,

$$
\|G\|=\left(2\left|G_{1}\right|-3\right)+\left(2\left|G_{2}\right|-3\right)-\|Q\|=(2|G|-3)+(2|Q|-3-\|Q\|)=2|G|-3 .
$$

Note that, as $Q$ is a clique, any stable cutset in $G$ is also a stable cutset in $G_{1}$ or $G_{2}$. Hence, by induction again, $G$ has no stable cutset.

Theorem 5. Let $G$ be a graph with $\|G\| \leqslant 2|G|-3$. Then $G$ contains a stable cutset or $G \in \mathcal{G}_{s c}$.

Proof. Our proof starts with a number of claims. For the sake of contradiction, we assume that $G$ is a minimal counterexample to Theorem 5. Then, by Theorem 1 ,

Claim 6. $\|G\|=2|G|-3$.

Claim 7. Every vertex $v$ lies in a triangle.

Otherwise, $N(v)$ would be a stable cutset in $G$.

Claim 8. $G$ contains no $K_{2}$-cutset and no $K_{3}$-cutset.

Otherwise, let $G$ contain a cutset $Q$ isomorphic to $K_{2}$ or $K_{3}$. Write $G=G_{1} \cup G_{2}$ with $G_{1} \cap G_{2}=Q$. Since $G$ has no stable cutset and $Q$ is a clique, $G_{1}$ and $G_{2}$ have no stable cutset. By Theorem 1, $\left\|G_{i}\right\| \geqslant 2\left|G_{i}\right|-3, i=1,2$, hence, by Claim 6, $\left\|G_{i}\right\|=2\left|G_{i}\right|-3$. Therefore, by the minimality of $G, G_{i} \in \mathcal{G}_{s c}$, and thus $G \in \mathcal{G}_{s c}$, a contradiction. 


\section{Claim 9. $G$ is 3-connected.}

Otherwise, by Claim 8, $G$ would contain a stable cutset.

Claim 10. G contains no 3-edge matching cut.

Otherwise, let $M=\left\{x_{1} y_{1}, x_{2} y_{2}, x_{3} y_{3}\right\}$ be a matching cut of $G$. Since $G$ is 2-connected, $G-M$ has exactly two components, say $G_{1}$ and $G_{2}$. Then the set of all edges between $G_{1}$ and $G_{2}$ is exactly $M$, and we may assume that $x_{1}, x_{2}, x_{3} \in V\left(G_{1}\right), y_{1}, y_{2}, y_{3} \in V\left(G_{2}\right)$. Now, if $\left\{x_{1}, x_{2}, x_{3}\right\}$ is not a clique, say $x_{1} x_{2} \notin E(G)$, then $\left\{x_{1}, x_{2}, y_{3}\right\}$ is a stable cutset of $G$, a contradiction. So, $\left\{x_{1}, x_{2}, x_{3}\right\}$ and, by symmetry, $\left\{y_{1}, y_{2}, y_{3}\right\}$ are cliques. Since $G \neq \overline{C_{6}}$, at least one of these cliques must be a cutset of $G$, contradicting Claim 8 .

Claim 11. G contains no $K_{4}^{-}$.

Otherwise, contract the edge between the two vertices of degree 3 in this (not necessarily induced) subgraph, resulting in a graph $G^{\prime}$. By Claim 9, $G^{\prime}$ is 2-connected. By Claim 6, $\left\|G^{\prime}\right\| \leqslant\|G\|-3=2|G|-6=2\left|G^{\prime}\right|-4$. By Theorem 2, $G^{\prime}$ contains a stable cutset not containing the new vertex, which is also a stable cutset in $G$.

Claim 12. For any two non-adjacent vertices $x$, y we have $|N(x) \cap N(y)| \leqslant 2$.

Otherwise, contract the two vertices, and get a (2-connected) graph $G^{\prime}$ with $\left\|G^{\prime}\right\| \leqslant$ $2\left|G^{\prime}\right|-4$. Then, $G^{\prime}$ has a stable cutset by Theorem 1 which yields a stable cutset in $G$.

Claim 13. $G$ contains no $P_{3}$-cutset.

Otherwise, let $\{x, y, z\}$ be a cutset of $G$ such that $x y, y z \in E(G)$, and let $G_{1}$ and $G_{2}$ be induced subgraphs of $G$ with $G=G_{1} \cup G_{2}$ and $G_{1} \cap G_{2}=\{x, y, z\}$. Then

$$
\left\|G_{1}\right\|+\left\|G_{2}\right\|=\|G\|+2=2|G|-1=2\left|G_{1}\right|+2\left|G_{2}\right|-7 .
$$

Thus, by symmetry $\left\|G_{1}\right\| \leqslant 2\left|G_{1}\right|-4$, and note that, by Claim $9, y$ is not a cut vertex of $G_{1}$. Therefore, by Corollary $3, G_{1}$ contains a stable cutset not containing $y$. But this is then also a stable cutset in $G$.

Claim 14. In every triangle, at least two vertices belong to other triangles as well.

Proof of Claim 14: Assume that $x y z$ is a triangle and $y$ and $z$ are in no other triangles. Then there is an edge $y^{\prime} z^{\prime}$ with $y^{\prime} \in N(y)$ and $z^{\prime} \in N(z)$ as otherwise, by Claim 11, $(N(y) \cup N(z)) \backslash\{y, z\}$ is a stable cutset. Contracting $\left\{y, z^{\prime}\right\}$ to a new vertex $v$ yields a graph $G^{\prime}$ with $\left\|G^{\prime}\right\| \leqslant 2\left|G^{\prime}\right|-3$. Since every stable cutset in $G^{\prime}$ yields a stable cutset in $G, G^{\prime}$ has no stable cutset. So $G^{\prime} \in \mathcal{G}_{s c}$ by the minimality of $G$.

Now assume that $G^{\prime}$ contains a 3-edge matching cut $M$. Then by Claim 10, $v$ is in one of the edges in $M$, say $M=\{a u, b v, c w\}$, and further, we have $b y^{\prime}, b z \in E(G)$. Let the two triangles in $G^{\prime}$ enclosing $M$ be $a b c$ and uvw, where $b \in\left\{y^{\prime}, z\right\}$ by Claim 12 .

Let $G_{1}$ be the component of $G-\left\{a u, b y^{\prime}, b z, c w\right\}$ containing $a b c$. By Claim $8, G_{1}=a b c$. Let $G_{2}=G-G_{1}$, and note that $\left\|G_{2}\right\|=2\left|G_{2}\right|-4$. Further, $G_{2}$ is 2-connected as any 1-cutset in $G_{2}$ would yield a 2-cutset in $G$ if we add $b$ to it. We may assume by symmetry that $z^{\prime} w \in E(G)$ (we will not use the fact that $y$ lies in only one triangle, so $y$ and $z^{\prime}$ are 
symmetric in the following argument). Further assume that $y u \in E(G)$. By Theorem 2, $G_{2}$ contains a stable cutset $X$ not containing $w$. As $X$ is not a stable cutset of $G, y$ is in a different component of $G_{2}-X$ than $w$. Thus, $u \in X$ and $X \cup\{b\}$ is a cutset of $G$, and as this is not a stable cutset, $z^{\prime} \in X$. But then $X \cup\{c\}$ is a stable cutset in $G$, a contradiction. So $y u \notin E(G)$ and therefore $z^{\prime} u \in E(G)$. By Theorem $2, G_{2}$ contains a stable cutset $X$ not containing $z^{\prime}$, with $y$ and $z^{\prime}$ in different components of $G_{2}-X$. But then $X \cup\{b\}$ is a stable cutset in $G$, a contradiction. Therefore, $G^{\prime}$ contains no 3-edge matching cut.

As a result, $G^{\prime}$ can be built by starting with a triangle and recursively glueing on triangles along an edge ( $G^{\prime}$ is a so-called 2-tree). As $G$ is 3-connected, every such 2-cutset in $G^{\prime}$ has the form $u v$, and $u$ is connected to exactly one of $y$ and $z^{\prime}$ by Claim 13 . Further, every vertex of degree at least 3 in $G^{\prime}$ lies in such a cutset.

On the other hand, there are at least two vertices of degree 2 in $G^{\prime}$. As $G$ is 3-connected, such vertices must lie in $N_{G}(y) \cap N_{G}\left(z^{\prime}\right)$, but by Claim $12, N_{G}(y) \cap N_{G}\left(z^{\prime}\right)=\left\{y^{\prime}, z\right\}$. Thus, exactly the two vertices $y^{\prime}$ and $z$ have degree 2 in $G^{\prime}$, and $N_{G}(z)=\left\{x, y, z^{\prime}\right\}$. By a symmetric argument using a contraction of $\left\{y^{\prime}, z\right\}$ instead of $\left\{y, z^{\prime}\right\}$ in the beginning, $N_{G}(y)=\left\{x, y^{\prime}, z\right\}$. But this implies that $N_{G}\left(z^{\prime}\right)=V(G) \backslash\left\{y, z^{\prime}\right\}$, as every vertex in $V(G) \backslash\left\{y, z^{\prime}\right\}$ is in $N_{G^{\prime}}(v)=N_{G}(y) \cup N_{G}\left(z^{\prime}\right)$. In particular, $x z^{\prime} \in E(G)$. This contradicts Claim 11, as $G\left[\left\{x, y, z, z^{\prime}\right\}\right]$ is then a $K_{4}^{-}$, hence Claim 14 follows.

Consider the vertex-triangle incidence graph $H$ of $G$, i.e., the bipartite graph with partite sets $V(G)$ and the set of all triangles $T(G)$ in $G$, with an edge between a vertex $v \in V(G)$ and a triangle $T \in T(G)$ if $v \in V(T)$. By Claim 14, $H$ is not a tree.

Let $x_{1} T_{1} x_{2} T_{2} \ldots x_{k} T_{k} x_{1}$ be a shortest cycle in $H$. By Claim 11, $H$ has no cycles of length less or equal to 6 , so $k \geqslant 4$. Then $C=x_{1} x_{2} \ldots x_{k} x_{1}$ is a cycle in $G$, and $V\left(T_{i}\right) \backslash V(C)$ consists of a distinct vertex for every $1 \leqslant i \leqslant k$.

If we contract $P=x_{1} x_{2} \ldots x_{k-1}$ to a new vertex $v$, we get a graph $G^{\prime}$ with $\left\|G^{\prime}\right\| \leqslant$ $2\left|G^{\prime}\right|-4$. If $v$ is not the unique cut vertex of $G^{\prime}$, then we can use Corollary 3 to find a stable cutset of $G^{\prime}$ not containing $v$, which is then also a stable cutset of $G$, a contradiction. Thus, $v$ is the unique cut vertex of $G^{\prime}$. Let $Y$ be a component of $G \backslash V(P)$ and $1 \leqslant r \leqslant s \leqslant k-1$, such that

$$
\left\{x_{r}, x_{s}\right\} \subseteq N(Y) \cap V(P) \subseteq\left\{x_{r}, \ldots, x_{s}\right\}
$$

and

$$
N(Z) \cap V(P) \backslash\left\{x_{r+1}, \ldots, x_{s-1}\right\} \neq \emptyset
$$

for all components $Z$ of $G \backslash V(P)$. Note that $s \geqslant r+2$ as $G$ is 3-connected. Now contract $x_{r+1} \ldots x_{s-1}$ to a new vertex $x$ and call the resulting graph $G^{\prime \prime}$. Then $\left\|G^{\prime \prime}\right\| \leqslant 2\left|G^{\prime \prime}\right|-3$, and let $G_{1}:=G^{\prime \prime}\left[Y \cup\left\{x_{r}, x, x_{s}\right\}\right]$ and $G_{2}:=G^{\prime \prime} \backslash Y$. As in Claim 13, we have

$$
\left\|G_{1}\right\|+\left\|G_{2}\right\|=\left\|G^{\prime \prime}\right\|+2 \leqslant 2\left|G^{\prime \prime}\right|-1=2\left|G_{1}\right|+2\left|G_{2}\right|-7 \text {. }
$$

Further, $x$ is neither a cut vertex of $G_{1}$ nor of $G_{2}$. Thus, by Corollary 3 , either $G_{1}$ or $G_{2}$ has a stable cutset not containing $x$. But this is also a stable cutset of $G$, a contradiction. 


\section{Complexity Issues}

With STABLE CUTSET we mean the following decision problem: 'Does a given graph admit a stable cutset?' The computational complexity of STABLE CUTSET has been addressed in a number research papers, e.g., [5, 1, 7, 8, 9]. To sum up, STABLE CUTSET is NP-complete for graphs of maximum degree five (even for $K_{4}$-free planar graphs with maximum degree five [8] and for 5-regular line graphs of bipartite graphs [9]), and is trivial for graphs of maximum degree three (by Theorem 1, such graphs with more than seven vertices always have a stable cutset). The complexity status of STABLE CUTSET is still open for graphs with maximum degree four.

By Theorem 1, STABLE CUTSET for graphs with maximum degree four remains open only in four cases, namely for graphs with $n$ vertices and $m$ edges where $2 n-3 \leqslant m \leqslant 2 n$.

Thus, the following problem is of interest and has been addressed in $[9,10]$ :

$\operatorname{StABle} \operatorname{Cutset}(n, m)$. Given a graph $G$ with $n$ vertices and $m$ edges. Does $G$ have a stable cutset?

It was shown in [9] that, for any given $\epsilon>0, \operatorname{stable} \operatorname{CUTSET}(n, m)$ is NP-complete for $m \geqslant(2+\epsilon) n$. By Theorem 1, $\operatorname{Stable} \operatorname{CUTSet}(n, m)$ is trivial for $m \leqslant 2 n-4$. By Theorem 5, we obtain the following:

Corollary 15. STABLE $\operatorname{CUTSET}(n, 2 n-3)$ is solvable in polynomial time.

Proof. Let $G$ be a graph with $n$ vertices and $m=2 n-3$ edges. Then, by Theorem 5 , $G$ has a stable cutset, or else $G$ must belong to $\mathcal{G}_{s c}$. Since the members of $\mathcal{G}_{s c}$ can be recognized in time $O\left(n^{4}\right)$ in an obvious way, Corollary 15 follows.

In fact, the recognition of $G \in \mathcal{G}_{s c}$ can be performed in quadratic time, based on the following observations. For every edge $x y$, we can in linear time test if $\{x, y\}$ is a cutset and determine the components of $G-\{x, y\}$. If $G \in \mathcal{G}_{s c}$, performing this for all $2 n-3$ edges, this process yields in quadratic time a set of at most $n-2$ components with at most a total of $3 n-6$ vertices, where each component is obtained from copies of $K_{3}$ and $\overline{C_{6}}$ via triangle identification. In particular, every vertex is in exactly one $K_{3}$. Further, every vertex of degree 2 is in a $K_{3}$-component, and the non-separating $K_{3}$ in other components consist exactly of the vertices of degree 3 . This way, we can easily recover the building blocks, the $\overline{C_{6}}$, used to build up the components in quadratic time, by cutting off one $\overline{C_{6}}$ which includes a non-separating $K_{3}$ at a time (linear time for each step, linear number of steps).

With a bit more effort, one can show that one can decide if $G \in \mathcal{G}_{s c}$ in time $O(n \log n)$, but for the sake of exposition we do not present the argument here. 


\section{References}

[1] A. Brandstädt, F. Dragan, V.B. Le, T. Szymczak, On stable cutsets in graphs, Discrete Appl. Math. 105 (2000) 39-50.

[2] Y. Caro, R. Yuster, Decomposition of slim graphs, Graphs Combinatorics 15 (1999) 5-19.

[3] G. Chen, R.J. Faudree, M.S. Jacobson, Fragile graphs with small independent cuts, J. Graph Theory 41 (2002) 327-341.

[4] G. Chen, X. Yu, A note on fragile graphs, Discrete Math. 249 (2002) 41-43.

[5] V. Chvátal, Recognizing decomposable graphs, J. Graph Theory 8 (1984) 51-53.

[6] D.G. Corneil, J. Fonlupt, Stable set bonding in perfect graphs and parity graphs, J. Combin. Theory (B) 59 (1993) 1-14.

[7] S. Klein, C.M.H. de Figueiredo, The NP-completeness of multi-partite cutset testing, Congressus Numerantium 119 (1996) 217-222.

[8] V.B. Le, R. Mosca, H. Müller, On stable cutsets in claw-free graphs and planar graphs, J. Discrete Algorithms 6 (2008) 256-276.

[9] V.B. Le, B. Randerath, On stable cutsets in line graphs, Theoret. Comput. Sci. 301 (2003) 463-475.

[10] "Selected open problems," In: 7th C5 Graph Theory Workshop, Kurort Rathen, 12.16.05.2003.

http://www . mathe.tu-freiberg.de/math/inst/theomath/WorkshopRathen.html

[11] A. Tucker, Coloring graphs with stable cutsets, J. Combin. Theory (B) 34 (1983) 258-267. 